\title{
Search Algorithms for Unstructured Peer-to-Peer Networks
}

\author{
Reza Dorrigiv \\ School of Computer Science \\ University of Waterloo \\ Waterloo, ON, N2L 3G1, Canada \\ Email: rdorrigiv@uwaterloo.ca
}

\author{
Alejandro López-Ortiz \\ School of Computer Science \\ University of Waterloo \\ Waterloo, ON, N2L 3G1, Canada \\ Email: alopez-o@uwaterloo.ca
}

\author{
Paweł Prałat \\ Department of Mathematics and Statistics \\ Dalhousie University \\ Halifax, NS, B3H 3J5, Canada \\ Email: pralat@mathstat.dal.ca
}

\begin{abstract}
We study the performance of several search algorithms on unstructured peer-to-peer networks, both using classic search algorithms such as flooding and random walk, as well as a new hybrid algorithm proposed in this paper. This hybrid algorithm first uses flooding to find sufficient number of nodes and then starts random walks from these nodes. We compare the performance of the search algorithms on several graphs corresponding to common topologies proposed for peerto-peer networks. In particular, we consider binomial random graphs, regular random graphs, power-law graphs, and clustered topologies. Our experiments show that for binomial random graphs and regular random graphs all algorithms have similar performance. For power-law graphs, flooding is effective for small number of messages, but for large number of messages our hybrid algorithm outperforms it. Flooding is ineffective for clustered topologies in which random walk is the best algorithm. For these topologies, our hybrid algorithm provides a compromise between flooding and random walk. We also compare the proposed hybrid algorithm with the $k$-walker algorithm on power-law and clustered topologies. Our experiments show that while they have close performance on clustered topologies, the hybrid algorithm has much better performance on power-law graphs. We theoretically prove that flooding is effective for regular random graphs which is consistent with our experimental results.
\end{abstract}

\section{INTRODUCTION}

Peer-to-peer networks are widely used for file sharing purposes. This type of usage tends to favour resilient, decentralized architectures over centralized solutions. However this comes at a penalty in ease of searching. At first, peerto-peer systems addressed this shortcoming by incorporating a flooding mechanism for resource discovery. A node in the peer-to-peer network broadcasts a query message to its neighbours. The neighbours in turn are responsible for reporting any matches as well as forwarding the message to its neighbours, if necessary. This mechanism has been proven effective in practice for finding items which are prevalent across the peer-to-peer network, but otherwise ineffective and resource consuming.

As a consequence numerous alternatives search techniques have been proposed, ranging from variants in flooding algorithms, to structured distributed solutions to centralized indices [3]. Each of these techniques has its own merits and drawbacks.

In this paper we consider searching for an object in an unstructured decentralized peer-to-peer networks such as
Gnutella or FastTrack. It is known that in this context flooding excels in the search of popular items which are widely available. On the other hand, traditional time-to-live (TTL) techniques fail to locate items that are not available locally. Gkantsidis et al. (INFOCOM'04) [4] studied random walks as an alternative to TTL-based flood techniques. They showed that certain types of random walks can outperform flooding techniques provided that certain modifications in the topology are introduced as part of the search. This work was further extended by the same authors [5] in INFOCOM'05. In that work, they consider the impact of a random walk followed by shallow flooding and observe that if we assume that edge criticality is known then the graph can be labeled in such a way as to obtain an effective search technique. A similar study is by Lv et al. [8] where they consider $k$ random walkers in random, power-law, grid and Gnutella graphs.

In this paper we systematically study the effect of random walk versus flooding in a variety of settings and topologies. We consider, in particular, binomial random graphs, $d$-regular graphs, power-law graphs and clustered topologies. We follow the methodology of Gkantsidis et al. [4], [5] in which a maximum number of messages is set and then we determine what percentage of the nodes in the peer-to-peer graph are visited using this number of messages. In a sense this is a measure of per-message effectiveness of the search method. As well, we consider the case in which changes to the topology are not feasible due to the unstructured nature of the peer-topeer topology.

We proceed to describe the search algorithms which are of interest in our work.

Flooding. Flooding is one of the simplest search strategies and yet one of the most commonly used in peer-to-peer networks. For instance, it is the search method employed by the Gnutella network. The search proceeds as follows: the origin of the search sends "discovery" messages to all its neighbours, which in turn propagate the message to their own neighbours (except the neighbour from which they have received the message) and so on.

Flooding as described above is unrestricted in the sense that there is no constraint on the number of messages generated by the search request. A simple way to limit the range of 
the flooding is to introduce the so-called time-to-live (TTL) parameter. More superficially, the origin of the search sends a discovery message to all its neighbours, along with the parameter TTL. The recipients decrease TTL by one and if TTL is still positive they propagate the message to their own neighbourhoods (except the neighbour from which they have received the message), and so fourth. In this way, it is guaranteed that the search is restricted within a ball of radius TTL from the origin of the search.

The popularity of flooding is mainly due to its simplicity; it is easy to implement and easy to support. Moreover, in its unrestricted version (or when TTL is set to a sufficiently large value) it will always succeed in the search. The most striking drawback of flooding is that it suffers from rather prohibitive message complexity.

Normalized Flooding Normalized flooding is the same as flooding, except that every node sends the message only to a subset of its neighbours. Let $\delta$ be the minimum degree (number of neighbours) of a node in the network. If a node has more than $\delta$ neighbours, then it sends the message only to $\delta$ nodes in its neighbourhood that are selected uniformly at random.

Simulation of Random Walks. Random walks have emerged in the area of peer-to-peer networks as alternatives to flooding. The idea is that instead of forwarding a request to all the neighbours, the recipient sends it to a random neighbour. The appealing property of the random walk method is the small message complexity, which means the algorithm scales well with the size of the network. In addition, random walks have been studied extensively from a theoretical point of view. Naturally, we are interested in the time (or, equivalently, on the number of hops) the random walk needs to locate the host sought. An efficient random walk will have the property that this time is small.

There are variants of the random walk method which aim to reduce the search time. One variant involves the use of $k$ independent random walks, all simulated in parallel from the origin. The search is successful once the file is located by any one of the individual random walks. We call this variant the $k$-walkers algorithm. A different variant introduces small local flooding into the random walk. More precisely, every node in the random walk initiates a (small) flooding, namely flooding with a small TTL value. This hybrid algorithm was proposed and studied in [5].

Local flooding with $k$ independent random walks. In this paper we propose and study a new hybrid algorithm, as a compromise between flooding and random walks. The idea is to first perform a (local) flooding starting from the origin of the search; the flooding continues until exactly $k$ new outer nodes have been discovered, for some predefined value of $k$. In the case where one of these nodes has the file, the search is successful and the origin is notified. Otherwise, each of the $k$ nodes initiates an independent random walk.
What is the motivation behind such a hybrid algorithm? Clearly, we are trying to exploit the positive characteristics of two widely different algorithms, namely flooding and random walks. If the file is located close to the origin, the local flooding would be sufficient to locate it fast, and with few messages exchanged. If the file is located away from the network, it is expected that it will be located by one of the random walks, but more importantly, since the flooding occurs only locally, the message complexity is small.

There exist situations in which one might anticipate that the two hybrid algorithms described above will not necessarily have similar performance. For instance consider a network which consists by several dense clusters (e.g., cliques) which are interconnected by means of a very sparse network (e.g., a tree that spans the representatives of each cluster). If we initiate a search from a certain cluster, assuming that the file is located in a different cluster, using random walks with local flooding, it may happen that the random walk remains "trapped" within the cluster of the origin of the search. On the other side, one would hope that local flooding combined with independent random walks may resolve this problem, since the flooding could discover vertices outside the origin's cluster at an earlier time.

\section{TheOreticAl RESUlts}

In this section we prove that in $d$-regular graphs, flooding finds new vertices most of the time. Similar behaviour can be proved for $G_{n, d / n}$. Therefore we expect flooding to have a good performance on these topologies. Let $N_{i}(u)$ denote the set of vertices at distance at most $i$ from $u$. Note that, in the early stages of the flooding process, the graph revealed from vertex $u$ tend to be a tree, that is, the number $n_{i}$ of elements in $N_{i}(u)$ is approximately $n_{i-1}+(d-1)\left(n_{i-1}-n_{i-2}\right)$. Thus, after $i \sim \frac{3}{4} \log _{d-1} n$ steps, we expect $N_{i}(u)$ to contain about $n^{3 / 4}$ vertices.

Let $f_{i}$ denote the total number of queries for flooding with $T T L=i$, that is, $f_{i}=d \sum_{j=0}^{i-1}(d-1)^{j}=\frac{d\left((d-1)^{i}-1\right)}{d-2}$, and $i_{0}=\frac{1}{2} \log _{d-1} n$. An event is said to hold with high probability (w.h.p.), if it holds with probability $1-o(1)$ as $n \rightarrow \infty$.

Lemma 1: Let $\omega(n)$ be any function of $n$ such that $\omega(n) \rightarrow$ $\infty$ as $n \rightarrow \infty$. For $i \leq i_{0}-\omega(n)$ w.h.p. the cardinality $n_{i}$ of $N_{i}(u)$ equals $f_{i}$. Moreover, for $i \leq i_{0}+\omega(n)$ w.h.p. $n_{i}=$ $f_{i}-O\left(\omega(n)(d-1)^{3\left(i-i_{0}\right)+\omega(n)}\right)$.

Proof: Note that the expected number of "cross edges" that generate a new cycle(s) at step $i+1$ is equal to $O\left(n_{i}^{2} / n\right)=$ $O\left(f_{i}^{2} / n\right)=O\left((d-1)^{2 i} / n\right)$.

Consider $i_{1}=\left\lfloor i_{0}-\omega(n)\right\rfloor=\left\lfloor\frac{1}{2} \log _{d-1} n-\omega(n)\right\rfloor$. The expected number of "cross edges" found up to time-step $i_{1}$ is equal to $\sum_{j=0}^{i_{1}-1} O\left((d-1)^{2 j} / n\right)=O\left((d-1)^{2 i_{1}} / n\right)=o(1)$. Thus, from Markov inequality, until step $i_{1}$ w.h.p. there are no "cross edges", hence w.h.p. $N_{i_{1}}(u)$ is a tree and $n_{i}=f_{i}$ for $i \leq i_{1}$.

Also, the expected number of "cross edges" added between step $i_{1}+1$ and step $i, i \leq\left\lfloor i_{0}+\omega(n)\right\rfloor=\left\lfloor\frac{1}{2} \log _{d-1} n+\omega(n)\right\rfloor$ is equal to $\sum_{j=i_{1}}^{i-1} O\left((d-1)^{2 j} / n\right)=O\left((d-1)^{2 i} / n\right)=$ 
$O\left((d-1)^{2\left(i-i_{0}\right)}\right)$. Thus, again from Markov inequality, w.h.p. the total number of "cross edges" at time $i$ is at most $O\left(\omega(n)(d-1)^{2\left(i-i_{0}\right)}\right)$. Since one "cross edge" added at this time interval can destroy tree branch of size $O((d-$ $\left.1)^{i-i_{0}+\omega(n)}\right)$, the following equality holds w.h.p. $n_{i}=f_{i}-$ $O\left(\omega(n)(d-1)^{2\left(i-i_{0}\right)}\right) \cdot O\left((d-1)^{i-i_{0}+\omega(n)}\right)$, which completes the proof of the theorem.

The next theorem is a simple consequence of the Lemma 1.

Theorem 1: Let $\hat{\omega}(n)$ be any function such that $\hat{\omega}(n) \rightarrow \infty$ as $n \rightarrow \infty$. For $i \leq \hat{i}=\frac{3}{4} \log _{d-1} n-\hat{\omega}(n)$ w.h.p. $\frac{n_{i}}{f_{i}}=$ $1-o(1)$. $i \leq \hat{i}$

Proof: Let $\omega(n)=o(\hat{\omega}(n))$. From Lemma 1, for any

$$
\begin{aligned}
\frac{n_{i}}{f_{i}} & =1-O\left(\omega(n)(d-1)^{2 i-3 i_{0}+\omega(n)}\right) \\
& =1-O\left(\omega(n)(d-1)^{-2 \hat{\omega}(n)+\omega(n)}\right) \\
& \left.=1-O(d-1)^{-2(1+o(1)) \hat{\omega}(n)}\right) \\
& =1-o(1)
\end{aligned}
$$

and the assertion follows.

\section{EXPERIMENTAL EVALUATION}

In this section we present the results of the experimental evaluation of our algorithms. Subsection III-A provides details concerning the generation of inputs and the performance metrics used. Subsection III-B is a discussion of the graph topologies we are studying. Finally in Subsection III-C we present the results we obtained through experiments along with a discussion of their significance. We implemented the algorithms in $\mathrm{C} / \mathrm{C}++$, and run them in a cluster of clusters (see Section $\mathrm{V}$ for more details). The program code can be found at the following address: "http://www.mathstat.dal.ca/ pralat/".

\section{A. Methodology}

For our experiments we generate 80 random graphs (for each topology) of 100,000 nodes each, and run the algorithms on each graph; this ensures that we do not, accidentally, choose an outlier graph. For each topology and each algorithm, we consider several aggregate functions (mean, median, standard deviation) on the results of running that algorithm on the corresponding 80 graphs.

On a similar note, for each of 80 input graphs, we consider every vertex as a possible origin for a search, and we evaluate the average performance of the algorithms over all origins (and all input graphs). This is particularly important for clustered topologies and power-law graphs in which such outlier vertices are not infrequent.

Naturally, we want to evaluate the algorithms on input graphs which are connected. However, the popular methods for randomly generating graphs (see Subsection III-B) do not necessarily produce connected graphs. We remedy this situation by the following process: First, we make every isolated vertex adjacent to a vertex in the graph, by adding an edge at random. Then, we find the connected components of the new graph (using BFS) and add a random edge between components. This guarantees the final graph is connected.

For a fair comparison of the various search algorithms, we require that they all use the same number of messages. For our experiments we look at values of $5^{5}, 5^{6}$, and $10^{5}$ for some topologies and $10^{4}$ and $10^{5}$ for the others.

The major performance metric we use is the vector $V=$ $\left(v_{0}, v_{1}, \ldots, v_{k}\right)$, where $v_{i}$ denotes the average number of nodes visited exactly $i$ times, over all searches. Clearly, the most significant coordinate of $V$ is $v_{0}$ since the number of distinct nodes visited during the execution of a search algorithm is $n-v_{0}$. An additional interpretation of the significance of $v_{0}$ is the following: Suppose $c$ copies of a file we seek are placed over the network, uniformly at random. Then we expect $\frac{n-v_{0}}{n} c$ copies of this file to be discovered by the search algorithm. The remaining coordinates of $V$ capture repetitive visits of nodes in the graph. Clearly, a good search algorithm would exhibit very small values of $v_{i}$ 's , especially for large values of $i$

\section{B. Topologies}

We focus on topologies which have been studied extensively in the past, and in particular for topologies for which there is good empirical evidence that they model, at least to a satisfactory level, the structure of modern communication networks. Among the topologies we study, the binomial random graph and the $d$-regular random graph models are models of mostly theoretical interest whereas power-law graphs and clustered topologies come closer to capturing the behaviour of realistic networks.

Binomial random graphs. Given a real number $p, 0 \leq p \leq 1$ and a collection of vertices $V$, a random binomial graph is generated by including, for every pair of vertices $v_{i}, v_{j} \in V$, an edge $\left(v_{i}, v_{j}\right)$ with probability $p$; the random experiment is repeated independently for all $\left(\begin{array}{l}n \\ 2\end{array}\right)$ pairs of vertices. We show the corresponding binomial random graph by $G_{n, p}$. This model has been studied extensively (see [6] for the definitive treatment of this topic). For instance, well-known theorems concerning this class of graphs characterize the threshold at which a giant component emerges or the random graph becomes connected.

For our evaluation, we are looking at graphs with expected degree 5 and 10.

$d$-regular random graphs. A $d$-regular graph is defined as a graph in which every vertex has degree $d$; a random $d$ regular graph is a graph chosen uniformly at random from the space of all $d$-regular graphs on $n$ vertices. Random $d$-regular graphs have certain appealing properties such as low diameter and good connectivity and, as in the case of binomial random graphs, have also been studied extensively from a theoretical point of view.

Generating a random $d$-regular graph is a subtle issue. One approach is the so called configurational model which provides a framework for creating a random graph with a certain degree 
sequence (see e.g., [12]). This approach is relatively simple, but has the drawback that it may yield a multigraph (i.e., multiedges and self-loops may be present) instead of a simple graph. Instead, we choose to implement the following $d$ process: we begin with $n$ isolated vertices, to which edges are added randomly one by one so that the maximum degree of the induced graph is always at most $d$. It is known [11] that with probability tending to 1 as $n \rightarrow \infty$, the result of this process is a $d$-regular graph (except for one vertex of degree $d-1$ when $d n$ is odd).

For our evaluation, we are looking at 5-regular and 10regular graphs.

Power-law graphs. In recent years substantial evidence has been presented that networks such as the Web graph exhibit a power-law with respect to the degrees of their nodes. More precisely, Broder et al. [2] noticed that the distribution of degrees in the Web graph follows a power-law: the fraction of vertices with degree $d$ is proportional to $d^{-\gamma}$, where $\gamma$ is a constant independent of the size of the network (more precisely, $\gamma \sim 2.1$ for in-degrees, $\gamma \sim 2.7$ for out-degrees). As a reminder, the web graph is defined as the graph in which every node corresponds to a static web page and for every hyperlink between two pages there exists a directed edge incident to the corresponding nodes.

There are several known models for the web graph (see for example the general survey [1]). We chose to implement a recently developed model, known as the Protean Model. Our choice is motivated by certain very attractive properties of such graphs: in particular, it has been shown (see [9]) that the protean graph has one giant component which contains a positive fraction of all vertices and is of diameter $\Theta(\log n)$. This indicates that the protean graph captures the structure of small-world networks (of which real-life examples are web graph and other natural networks). Note also that the definition of protean process allows us to study the recovery time; an interesting and very important property which does not have its counterpart for the other models.

The formal definition of the protean graph $\mathcal{P}_{n}(d, \eta)$ is somewhat technical (we refer the reader to [7] for more details; see also [10] for extended version of standard protean graph). Nevertheless the idea behind its definition is simple. This model takes into account an additional natural parameter of a vertex, its age, and predicts how it influences the degree of a vertex. We start with any graph $G$ with vertex set $[n]$, and at each time-step we pick randomly one of the vertices $v$ to be renewed. More precisely, we delete from $G$ all edges incident to $v$; this corresponds to a removal of a random node from the network. Then we generate $d$ new edges from $v$ to existing vertices chosen randomly with weighted probabilities ('old' vertices have bigger probability of being chosen). Note that vertex $v$ can be viewed as a new node which establishes connection with some nodes in the network. When all vertices are renewed at least once, the random graph is a protean graph $\mathcal{P}_{n}(d, \eta)$.

In [7] it is shown that the degrees of the $\mathcal{P}_{n}(d, \eta)$ are
TABLE I

EXPERIMENTAL RESULTS FOR $G_{n, p}$ WITH $n=100,000$ AND $p=5 / n$

\begin{tabular}{|c|l|c|c|c|c|c|}
\hline $\mathrm{A}$ & $\mathrm{B}$ & Mean & Perc. of best & Minimum & Maximum & $\sigma$ \\
\hline $5^{5}$ & $\mathcal{F}$ & 3072.177 & 100.000 & 3071.768 & 3072.548 & 0.137 \\
$5^{5}$ & $\mathcal{N} \mathcal{F}$ & 3066.836 & 99.826 & 3066.157 & 3067.383 & 0.238 \\
$5^{5}$ & $\mathcal{R} \mathcal{W}$ & 3034.931 & 98.788 & 3032.454 & 3036.592 & 0.718 \\
$5^{5}$ & $\mathcal{H}_{1}$ & 3035.026 & 98.791 & 3032.569 & 3036.718 & 0.728 \\
$5^{5}$ & $\mathcal{H}_{2}$ & 3035.282 & 98.799 & 3032.854 & 3036.947 & 0.697 \\
$5^{5}$ & $\mathcal{H}_{3}$ & 3046.035 & 99.149 & 3044.455 & 3047.162 & 0.490 \\
\hline $5^{6}$ & $\mathcal{F}$ & 14215.424 & 99.811 & 14209.195 & 14222.816 & 2.854 \\
$5^{6}$ & $\mathcal{N} \mathcal{F}$ & 14242.398 & 100.000 & 14237.605 & 14246.279 & 1.932 \\
$5^{6}$ & $\mathcal{R} \mathcal{W}$ & 14120.694 & 99.145 & 14107.459 & 14129.181 & 3.885 \\
$5^{6}$ & $\mathcal{H}_{1}$ & 14120.759 & 99.146 & 14107.630 & 14129.214 & 3.875 \\
$5^{6}$ & $\mathcal{H}_{2}$ & 14121.114 & 99.148 & 14107.901 & 14129.619 & 3.854 \\
$5^{6}$ & $\mathcal{H}_{3}$ & 14122.017 & 99.155 & 14108.917 & 14130.281 & 3.774 \\
\hline$n$ & $\mathcal{F}$ & 58867.496 & 99.231 & 58784.255 & 58936.885 & 30.072 \\
$n$ & $\mathcal{N} \mathcal{F}$ & 59323.840 & 100.000 & 59248.742 & 59383.612 & 23.729 \\
$n$ & $\mathcal{R \mathcal { W }}$ & 59297.404 & 99.955 & 59218.167 & 59351.545 & 25.516 \\
$n$ & $\mathcal{H}_{1}$ & 59297.513 & 99.956 & 59217.602 & 59351.357 & 25.495 \\
$n$ & $\mathcal{H}_{2}$ & 59297.580 & 99.956 & 59218.256 & 59351.393 & 25.468 \\
$n$ & $\mathcal{H}_{3}$ & 59298.196 & 99.957 & 59217.733 & 59352.119 & 25.401 \\
\hline
\end{tabular}

distributed according to a power-law. More precisely, the number of vertices of degree $k$ decreases roughly as $k^{-1-1 / \eta}$.

Clustered Topologies. These graphs are intended to model realistic networks which consist of relatively isolated clusters of nodes, connected through a backbone network. In particular, for our experiments we consider three models for clusters:

- $K_{l}$, i.e. clique of size $l$

- Random graph $G_{l, 1 / 2}$

- Random graph $G_{l, 1 / 5}$

Every cluster has a representative; we then connect the representatives by means of a 3-regular random graph. We consider clusters of size $l=100$.

\section{Experiments}

Tables I-XI show the Mean/Minimum/Maximum of the average number of distinct nodes that are discovered by each algorithm on the 80 graphs produced according to different topologies. In each table, the first column (labeled A) shows the number of messages and the second column (labeled B) denotes the algorithm. The algorithms are denoted as follows: flooding by $\mathcal{F}$, normalized flooding by $\mathcal{N F}$, random walk by $\mathcal{R W}$, and hybrid algorithm with $10^{i}$ random walkers by $\mathcal{H}_{i}$. For each topology and a fixed number of messages the row that corresponds to the algorithm with maximum Mean is highlighted. Also the percentage of Mean of other algorithms to this algorithm's Mean is shown. The other columns show the Mean, Minimum, Maximum, and standard deviation.

a) Analysis of results for binomial random graphs: Tables I and II show the results for binomial random graphs. We observe that in this case all algorithms have similar performance. Also as the number of messages increases, the performance of flooding decreases; it is the best algorithm for $5^{5}$ messages, but not for $N$ messages. We note that $\mathcal{R} \mathcal{W}, \mathcal{H}_{1}$, $\mathcal{H}_{2}$, and $\mathcal{H}_{3}$ behave almost the same.

b) Analysis of results for d-regular random graphs: Tables III and IV show the results for $d$-regular random graphs. Again the performances are very close. The good performance of flooding on these graphs is consistent with theoretical 
TABLE II

EXPERIMENTAL RESULTS FOR $G_{n, p}$ WITH $n=100,000$ AND $p=10 / n$

\begin{tabular}{|c|l|c|c|r|r|r|}
\hline $\mathrm{A}$ & $\mathrm{B}$ & Mean & Perc. of best & Minimum & Maximum & $\sigma$ \\
\hline $10^{4}$ & $\mathcal{F}$ & 9468.841 & 99.968 & 9467.126 & 9470.258 & 0.618 \\
$10^{4}$ & $\mathcal{N F}$ & 9471.838 & 100.000 & 9471.162 & 9472.433 & 0.282 \\
$10^{4}$ & $\mathcal{R W}$ & 9470.951 & 99.991 & 9470.313 & 9471.552 & 0.263 \\
$10^{4}$ & $\mathcal{H}_{1}$ & 9470.944 & 99.991 & 9470.304 & 9471.557 & 0.275 \\
$10^{4}$ & $\mathcal{H}_{2}$ & 9470.978 & 99.991 & 9470.453 & 9471.598 & 0.261 \\
$10^{4}$ & $\mathcal{H}_{3}$ & 9471.172 & 99.993 & 9470.518 & 9471.796 & 0.298 \\
\hline$n$ & $\mathcal{F}$ & 61209.108 & 99.707 & 61179.880 & 61234.376 & 12.791 \\
$n$ & $\mathcal{N F}$ & 61386.856 & 99.997 & 61365.743 & 61405.355 & 9.185 \\
$n$ & $\mathcal{R W}$ & 61388.751 & 100.000 & 61367.928 & 61407.741 & 9.038 \\
$n$ & $\mathcal{H}_{1}$ & 61388.728 & 100.000 & 61367.282 & 61408.125 & 9.062 \\
$n$ & $\mathcal{H}_{2}$ & 61388.819 & 100.000 & 61367.340 & 61407.824 & 9.110 \\
$n$ & $\mathcal{H}_{3}$ & 61388.855 & 100.000 & 61367.372 & 61408.264 & 9.003 \\
\hline
\end{tabular}

TABLE III

EXPERIMENTAL RESULTS FOR 5-REGULAR GRAPHS WITH $n=100,000$

\begin{tabular}{|c|l|c|c|r|r|c|}
\hline A & B & Mean & Perc. of best & Minimum & Maximum & $\sigma$ \\
\hline $5^{5}$ & $\mathcal{F}$ & 3077.056 & 100.000 & 3076.313 & 3077.660 & 0.266 \\
$5^{5}$ & $\mathcal{N F}$ & 3077.056 & 100.000 & 3076.313 & 3077.660 & 0.266 \\
\hline $5^{5}$ & $\mathcal{R} \mathcal{W}$ & 3076.770 & 99.991 & 3076.686 & 3076.867 & 0.038 \\
$5^{5}$ & $\mathcal{H}_{1}$ & 3076.768 & 99.991 & 3076.676 & 3076.839 & 0.034 \\
$5^{5}$ & $\mathcal{H}_{2}$ & 3076.776 & 99.991 & 3076.682 & 3076.856 & 0.034 \\
$5^{5}$ & $\mathcal{H}_{3}$ & 3076.974 & 99.997 & 3076.426 & 3077.435 & 0.204 \\
\hline $5^{6}$ & $\mathcal{F}$ & 14467.513 & 100.000 & 14462.400 & 14471.077 & 1.719 \\
$5^{6}$ & $\mathcal{N} \mathcal{F}$ & 14467.513 & 100.000 & 14462.400 & 14471.077 & 1.719 \\
$5^{6}$ & $\mathcal{R W}$ & 14465.866 & 99.989 & 14465.449 & 14466.116 & 0.132 \\
$5^{6}$ & $\mathcal{H}_{1}$ & 14465.865 & 99.989 & 14107.630 & 14129.214 & 3.875 \\
$5^{6}$ & $\mathcal{H}_{2}$ & 14465.888 & 99.989 & 14465.457 & 14466.261 & 0.152 \\
$5^{6}$ & $\mathcal{H}_{3}$ & 14466.101 & 99.990 & 14465.279 & 14466.774 & 0.304 \\
\hline$n$ & $\mathcal{F}$ & 63218.463 & 100.000 & 63198.744 & 63234.677 & 7.315 \\
$n$ & $\mathcal{N F}$ & 63218.463 & 100.000 & 63198.744 & 63234.677 & 7.315 \\
$n$ & $\mathcal{R} \mathcal{W}$ & 63213.218 & 99.992 & $63,212.362$ & $63,214.303$ & 0.413 \\
$n$ & $\mathcal{H}_{1}$ & 63213.175 & 99.992 & 63212.390 & 63213.939 & 0.374 \\
$n$ & $\mathcal{H}_{2}$ & 63213.080 & 99.991 & 63211.912 & 63213.840 & 0.440 \\
$n$ & $\mathcal{H}_{3}$ & 63213.291 & 99.992 & 63212.235 & 63214.413 & 0.487 \\
\hline
\end{tabular}

TABLE IV

EXPERIMENTAL RESULTS FOR 10-REGULAR GRAPHS WITH $n=100,000$

\begin{tabular}{|c|l|c|c|c|c|c|}
\hline $\mathrm{A}$ & $\mathrm{B}$ & Mean & Perc. of best & Minimum & Maximum & $\sigma$ \\
\hline $10^{4}$ & $\mathcal{F}$ & 9517.860 & 100.000 & 9516.843 & 9519.056 & 0.517 \\
\hline $10^{4}$ & $\mathcal{N} \mathcal{F}$ & 9516.986 & 99.991 & 9516.675 & 9517.306 & 0.151 \\
$10^{4}$ & $\mathcal{R} \mathcal{W}$ & 9516.496 & 99.986 & 9516.257 & 9516.649 & 0.075 \\
$10^{4}$ & $\mathcal{H}_{1}$ & 9516.515 & 99.986 & 9516.329 & 9516.669 & 0.075 \\
$10^{4}$ & $\mathcal{H}_{2}$ & 9516.513 & 99.986 & 9516.314 & 9516.685 & 0.081 \\
$10^{4}$ & $\mathcal{H}_{3}$ & 9516.674 & 99.988 & 9516.395 & 9516.981 & 0.117 \\
\hline$n$ & $\mathcal{F}$ & 63219.926 & 100.000 & 63212.771 & 63228.066 & 3.277 \\
$n$ & $\mathcal{N} \mathcal{F}$ & 63215.058 & 99.992 & 63213.303 & 63216.856 & 0.706 \\
$n$ & $\mathcal{R W}$ & 63213.002 & 99.989 & 63212.023 & 63213.810 & 0.431 \\
$n$ & $\mathcal{H}_{1}$ & 63213.065 & 99.989 & 63212.228 & 63213.905 & 0.364 \\
$n$ & $\mathcal{H}_{2}$ & 63213.050 & 99.989 & 63211.844 & 63213.721 & 0.347 \\
$n$ & $\mathcal{H}_{3}$ & 63213.106 & 99.989 & 63211.802 & 63213.884 & 0.419 \\
\hline
\end{tabular}

results of Section II. We observe that the standard deviations of $\mathcal{F}$ and $\mathcal{N F}$ is worse (larger) than other algorithms.

c) Analysis of results for power-law graphs with $\lambda=$ 2.1: Tables V and VI show the results for power-law graphs with $\lambda=2.1$. For small number of messages, flooding is the best algorithm according to Mean. As the number of messages increases, the efficiency of flooding decreases. For example it is the worst algorithm when we use $N$ messages. The standard deviation of flooding is much larger than other algorithms.

d) Analysis of results for power-law graphs with $\lambda=2.7$ : Tables VII and VIII show the results for power-law graphs with $\lambda=2.7$. For average degree $5, \mathcal{N F}$ is the best algorithm and for average degree $10, \mathcal{H}_{3}$ is the best algorithm. Flooding is the worst algorithm for large number of messages, that is, $N$ messages. Flooding has the largest standard deviation.

e) Analysis of results for clustered graphs: Tables IX, X, and XI show the results for clustered graphs. In general, all
TABLE V

EXPERIMENTAL RESULTS FOR POWER-LAW GRAPHS WITH $\lambda=2.1$ AND AVERAGE DEGREE 5

\begin{tabular}{|c|c|c|c|c|c|c|}
\hline $\mathrm{A}$ & $\mathrm{B}$ & Mean & Perc. of best & Minimum & Maximum & $\sigma$ \\
\hline $5^{5}$ & $\mathcal{F}$ & 2987.723 & 100.000 & 2950.868 & 3015.776 & 14.893 \\
\hline $5^{5}$ & $\mathcal{N} \mathcal{F}$ & 2727.960 & 91.306 & 3076.313 & 3077.660 & 0.266 \\
\hline $5^{5}$ & $\mathcal{R W}$ & 2571.427 & 86.066 & 2545.339 & 2606.159 & 15.165 \\
\hline $5^{5}$ & $\mathcal{H}_{1}$ & 2574.199 & 86.159 & 2546.333 & 2611.409 & 16.086 \\
\hline $5^{5}$ & $\mathcal{H}_{2}$ & 2585.539 & 86.539 & 2558.693 & 2621.724 & 15.446 \\
\hline $5^{5}$ & $\mathcal{H}_{3}$ & 2724.836 & 91.201 & 2709.105 & 2748.038 & 8.242 \\
\hline $5^{6}$ & $\mathcal{F}$ & 12851.139 & 100.000 & 12311.150 & 13398.720 & 291.761 \\
\hline $5^{6}$ & $\mathcal{N} \mathcal{F}$ & 11930.450 & 92.836 & 14462.400 & 14471.077 & 1.719 \\
\hline $5^{6}$ & $\mathcal{R W}$ & 11284.211 & 87.807 & 11183.438 & 11400.340 & 51.740 \\
\hline $5^{6}$ & $\mathcal{H}_{1}$ & 11289.183 & 87.846 & 11184.395 & 11404.517 & 54.289 \\
\hline $5^{6}$ & $\mathcal{H}_{2}$ & 11303.555 & 87.958 & 11198.152 & 11418.896 & 54.056 \\
\hline $5^{6}$ & $\mathcal{H}_{3}$ & 11414.221 & 88.819 & $1131^{\circ}$ & 11519.615 & 47.704 \\
\hline$n$ & $\mathcal{F}$ & 39074.911 & 76.818 & 35153.269 & 42861.851 & 1968.823 \\
\hline$n$ & $\mathcal{N F}$ & 50866.582 & 100.000 & 50677.829 & 51087.124 & 98.176 \\
\hline$n$ & $\mathcal{R W}$ & 48771.353 & 95.881 & 486 & 4893 & 60.500 \\
\hline$n$ & $\mathcal{H}_{1}$ & 48774.005 & 95.886 & 48658.002 & 4893 & 60.433 \\
\hline$n$ & $\mathcal{H}_{2}$ & 48789.317 & 95.916 & 48665.956 & 48950.913 & 61.582 \\
\hline$n$ & $\mathcal{H}_{3}$ & 48826.519 & 95.989 & 48703.350 & 48974.728 & 60.341 \\
\hline
\end{tabular}

TABLE VI

EXPERIMENTAL RESULTS FOR POWER-LAW GRAPHS WITH $\lambda=2.1$ AND AVERAGE DEGREE 10

\begin{tabular}{|c|c|c|c|c|c|c|}
\hline A & B & Mean & Perc. of best & Minimum & Maximum & $\sigma$ \\
\hline $10^{4}$ & $\mathcal{F}$ & 9297.479 & 100.000 & 8744.323 & 9557.168 & 141.577 \\
\hline $10^{4}$ & $\mathcal{N} \mathcal{F}$ & 7842.801 & 84.354 & 7691.151 & 8054.712 & 67.750 \\
\hline $10^{4}$ & $\mathcal{R W}$ & 7786.536 & 83.749 & 7638.957 & 7992.578 & 65.924 \\
\hline $10^{4}$ & $\mathcal{H}_{1}$ & 7786.141 & 83.745 & 7638.686 & 7992.406 & 65.904 \\
\hline $10^{4}$ & $\mathcal{H}_{2}$ & 7793.210 & 83.821 & 7647.361 & 7997.023 & 65.140 \\
\hline $10^{4}$ & $\mathcal{H}_{3}$ & 7891.596 & 79 & 776 & 036 & 57.312 \\
\hline$n$ & $\mathcal{F}$ & 48126 & 19 & 45706 & 50530.164 & 958.234 \\
\hline$n$ & $\mathcal{N} \mathcal{F}$ & 50295.169 & 99.718 & 49877.013 & 50867.788 & 195.987 \\
\hline$n$ & $\mathcal{R W}$ & 50409.017 & 99.944 & 50077.339 & 50897.000 & 164.873 \\
\hline$n$ & $\mathcal{H}_{1}$ & 50408 & & 500 & 508 & 164.818 \\
\hline$n$ & $\mathcal{H}_{2}$ & 5040 & 99 & 5007 & 5089 & 164.386 \\
\hline$n$ & $\mathcal{H}_{3}$ & 50437.379 & 100.000 & 50114.404 & 50917.094 & 161.747 \\
\hline
\end{tabular}

TABLE VII

EXPERIMENTAL RESULTS FOR POWER-LAW GRAPHS WITH $\lambda=2.7$ AND AVERAGE DEGREE 5

\begin{tabular}{|l|l|c|c|r|r|r|}
\hline $\mathrm{A}$ & $\mathrm{B}$ & Mean & Perc. of best & Minimum & Maximum & $\sigma$ \\
\hline $5^{5}$ & $\mathcal{F}$ & 3011.315 & 99.738 & 3005.965 & 3022.554 & 3.308 \\
$5^{5}$ & $\mathcal{N} \mathcal{F}$ & 3019.211 & 100.000 & 3008.989 & 3026.991 & 3.596 \\
$5^{5}$ & $\mathcal{R} \mathcal{W}$ & 2980.109 & 98.705 & 2969.928 & 2987.853 & 3.372 \\
$5^{5}$ & $\mathcal{H}_{1}$ & 2980.191 & 98.708 & 2969.948 & 2987.954 & 3.377 \\
$5^{5}$ & $\mathcal{H}_{2}$ & 2982.111 & 98.771 & 2972.243 & 2989.603 & 3.276 \\
$5^{5}$ & $\mathcal{H}_{3}$ & 3003.378 & 99.476 & 2997.927 & 3008.582 & 2.309 \\
\hline $5^{6}$ & $\mathcal{F}$ & 12793.147 & 93.138 & 12475.974 & 13190.481 & 153.404 \\
$5^{6}$ & $\mathcal{N} \mathcal{F}$ & 13735.759 & 100.000 & 13679.750 & 13781.684 & 20.349 \\
$5^{6}$ & $\mathcal{R W}$ & 13534.701 & 98.536 & 13478.217 & 13580.439 & 20.018 \\
$5^{6}$ & $\mathcal{H}_{1}$ & 13534.736 & 98.537 & 13478.076 & 13580.664 & 20.042 \\
$5^{6}$ & $\mathcal{H}_{2}$ & 13538.208 & 98.562 & 13482.125 & 13583.686 & 19.938 \\
$5^{6}$ & $\mathcal{H}_{3}$ & 13567.524 & 98.775 & 13518.301 & 13609.670 & 18.139 \\
\hline$n$ & $\mathcal{F}$ & 46328.091 & 81.009 & 44077.817 & 49146.477 & 1150.080 \\
\hline$n$ & $\mathcal{N F}$ & 57188.476 & 100.000 & 57081.934 & 57304.162 & 45.304 \\
$n n$ & $\mathcal{R} \mathcal{W}$ & 56648.461 & 99.056 & 56526.905 & 56767.528 & 51.876 \\
$n$ & $\mathcal{H}_{1}$ & 56648.330 & 99.055 & 56527.328 & 56767.581 & 51.869 \\
$n$ & $\mathcal{H}_{2}$ & 56650.912 & 99.060 & 56530.570 & 56770.165 & 51.712 \\
$n$ & $\mathcal{H}_{3}$ & 56678.879 & 99.109 & 56563.200 & 56795.721 & 50.489 \\
\hline
\end{tabular}

TABLE VIII

EXPERIMENTAL RESULTS FOR POWER-LAW GRAPHS WITH $\lambda=2.7$ AND AVERAGE DEGREE 10

\begin{tabular}{|c|l|c|c|r|r|r|}
\hline $\mathrm{A}$ & $\mathrm{B}$ & Mean & Perc. of best & Minimum & Maximum & $\sigma$ \\
\hline $10^{4}$ & $\mathcal{F}$ & 9124.481 & 99.661 & 9068.833 & 9168.929 & 21.113 \\
$10^{4}$ & $\mathcal{N F}$ & 9128.352 & 99.704 & 9093.659 & 9156.773 & 12.420 \\
$10^{4}$ & $\mathcal{R W}$ & 9125.727 & 99.675 & 9091.333 & 9153.804 & 12.458 \\
$10^{4}$ & $\mathcal{H}_{1}$ & 9125.678 & 99.675 & 9091.241 & 9153.888 & 12.460 \\
$10^{4}$ & $\mathcal{H}_{2}$ & 9127.180 & 99.691 & 9092.927 & 9155.113 & 12.398 \\
$10^{4}$ & $\mathcal{H}_{3}$ & 9155.475 & 100.000 & 9125.139 & 9180.442 & 10.894 \\
\hline$n$ & $\mathcal{F}$ & 50858.047 & 87.630 & 49452.064 & 52578.884 & 758.606 \\
$n$ & $\mathcal{N F}$ & 58020.466 & 99.971 & 57883.655 & 58121.120 & 49.104 \\
$n$ & $\mathcal{R W}$ & 58005.910 & 99.946 & 57870.928 & 58105.437 & 49.140 \\
$n$ & $\mathcal{H}_{1}$ & 58005.785 & 99.946 & 57870.597 & 58104.735 & 49.115 \\
$n$ & $\mathcal{H}_{2}$ & 58006.970 & 99.948 & 57872.266 & 58106.013 & 49.120 \\
$n$ & $\mathcal{H}_{3}$ & 58037.065 & 100.000 & 57904.662 & 58134.364 & 48.071 \\
\hline
\end{tabular}


TABLE IX

EXPERIMENTAL RESULTS FOR CLUSTERED GRAPHS THAT HAVE CLIQUES AS THEIR CLUSTERS

\begin{tabular}{|l|l|c|c|r|r|r|}
\hline $\mathrm{A}$ & $\mathrm{B}$ & \multicolumn{1}{|c|}{ Mean } & Perc. of best & Minimum & Maximum & $\sigma$ \\
\hline $5^{5}$ & $\mathcal{F}$ & 100.030 & 56.911 & 100.030 & 100.030 & 0.000 \\
$5^{5}$ & $\mathcal{N F}$ & 102.935 & 58.563 & 102.870 & 103.034 & 0.030 \\
$5^{5}$ & $\mathcal{R \mathcal { W }}$ & 175.767 & 100.000 & 175.311 & 176.331 & 0.211 \\
$5^{5}$ & $\mathcal{H}_{1}$ & 157.599 & 89.663 & 157.261 & 158.017 & 0.174 \\
$5^{5}$ & $\mathcal{H}_{2}$ & 112.824 & 64.190 & 112.701 & 112.938 & 0.049 \\
$5^{5}$ & $\mathcal{H}_{3}$ & 101.041 & 57.486 & 101.032 & 101.054 & 0.004 \\
\hline $5^{6}$ & $\mathcal{F}$ & 106.030 & 25.259 & 106.030 & 106.030 & 0.000 \\
$5^{6}$ & $\mathcal{N} \mathcal{F}$ & 113.059 & 26.934 & 112.899 & 113.206 & 0.070 \\
$5^{6}$ & $\mathcal{R W}$ & 419.766 & 100.000 & 418.520 & 420.919 & 0.520 \\
$5^{6}$ & $\mathcal{H}_{1}$ & 366.124 & 87.221 & 365.302 & 367.044 & 0.353 \\
$5^{6}$ & $\mathcal{H}_{2}$ & 263.615 & 62.800 & 263.009 & 264.106 & 0.222 \\
$5^{6}$ & $\mathcal{H}_{3}$ & 129.970 & 30.962 & 129.859 & 130.110 & 0.047 \\
\hline$n$ & $\mathcal{F}$ & 106.030 & 6.147 & 106.030 & 106.030 & 0.000 \\
$n$ & $\mathcal{N F}$ & 165.639 & 9.603 & 165.320 & 165.956 & 0.136 \\
$n$ & $\mathcal{R \mathcal { W }}$ & 1724.959 & 100.000 & 1718.010 & 1730.641 & 2.469 \\
$n$ & $\mathcal{H}_{1}$ & 1268.211 & 73.521 & 1263.732 & 1271.687 & 1.674 \\
$n$ & $\mathcal{H}_{2}$ & 614.545 & 35.627 & 613.530 & 615.662 & 0.434 \\
$n$ & $\mathcal{H}_{3}$ & 418.431 & 24.257 & 418.201 & 418.762 & 0.115 \\
\hline
\end{tabular}

TABLE X

EXPERIMENTAL RESUlts For Clustered Graphs that HAVE $G_{100,1 / 2}$ AS THEIR CLUSTERS

\begin{tabular}{|c|l|c|c|r|r|c|}
\hline $\mathrm{A}$ & $\mathrm{B}$ & \multicolumn{1}{|c|}{ Mean } & Perc. of best & Minimum & Maximum & $\sigma$ \\
\hline $5^{5}$ & $\mathcal{F}$ & 103.031 & 43.716 & 103.004 & 103.058 & 0.011 \\
$5^{5}$ & $\mathcal{N F}$ & 105.593 & 44.803 & 105.485 & 105.718 & 0.047 \\
$5^{5}$ & $\mathcal{R \mathcal { W }}$ & 235.685 & 100.000 & 234.990 & 236.534 & 0.324 \\
$5^{5}$ & $\mathcal{H}_{1}$ & 203.065 & 86.159 & 202.585 & 203.573 & 0.221 \\
$5^{5}$ & $\mathcal{H}_{2}$ & 124.482 & 52.817 & 124.381 & 124.638 & 0.051 \\
$5^{5}$ & $\mathcal{H}_{3}$ & 101.830 & 43.206 & 101.819 & 101.842 & 0.005 \\
\hline $5^{6}$ & $\mathcal{F}$ & 104.499 & 15.740 & 104.481 & 104.517 & 0.008 \\
$5^{6}$ & $\mathcal{N} \mathcal{F}$ & 123.664 & 18.626 & 123.361 & 123.955 & 0.103 \\
$5^{6}$ & $\mathcal{R W}$ & 663.923 & 100.000 & 662.021 & 665.353 & 0.709 \\
$5^{6}$ & $\mathcal{H}_{1}$ & 530.968 & 79.974 & 530.004 & 532.036 & 0.457 \\
$5^{6}$ & $\mathcal{H}_{2}$ & 349.951 & 52.710 & 349.500 & 350.451 & 0.191 \\
$5^{6}$ & $\mathcal{H}_{3}$ & 154.479 & 23.268 & 154.316 & 154.690 & 0.073 \\
\hline$n$ & $\mathcal{F}$ & 107.441 & 3.407 & 107.231 & 107.754 & 0.106 \\
$n$ & $\mathcal{N F}$ & 213.300 & 6.763 & 212.950 & 213.704 & 0.158 \\
$n$ & $\mathcal{R \mathcal { W }}$ & 3153.820 & 100.000 & 3138.785 & 3166.954 & 5.562 \\
$n$ & $\mathcal{H}_{1}$ & 2349.913 & 74.510 & 2337.288 & 2359.542 & 4.724 \\
$n$ & $\mathcal{H}_{2}$ & 951.127 & 30.158 & 948.444 & 953.171 & 0.999 \\
$n$ & $\mathcal{H}_{3}$ & 497.740 & 15.782 & 497.048 & 498.619 & 0.318 \\
\hline
\end{tabular}

TABLE XI

EXPERIMENTAL RESUlts FOR Clustered GRAPHS THAT HAVE $G_{100,1 / 5}$ AS THEIR CLUSTERS

\begin{tabular}{|c|l|r|c|r|r|r|}
\hline $\mathrm{A}$ & $\mathrm{B}$ & \multicolumn{1}{|c|}{ Mean } & Perc. of best & Minimum & Maximum & $\sigma$ \\
\hline $5^{5}$ & $\mathcal{F}$ & 103.110 & 27.427 & 103.082 & 103.137 & 0.013 \\
$5^{5}$ & $\mathcal{N} \mathcal{F}$ & 112.424 & 29.904 & 112.263 & 112.648 & 0.073 \\
$5^{5}$ & $\mathcal{R} \mathcal{W}$ & 375.948 & 100.000 & 375.029 & 377.084 & 0.453 \\
$5^{5}$ & $\mathcal{H}_{1}$ & 303.682 & 80.778 & 302.964 & 304.491 & 0.282 \\
$5^{5}$ & $\mathcal{H}_{2}$ & 153.582 & 40.852 & 153.335 & 153.787 & 0.098 \\
$5^{5}$ & $\mathcal{H}_{3}$ & 107.919 & 28.706 & 107.820 & 107.998 & 0.036 \\
\hline $5^{6}$ & $\mathcal{F}$ & 125.272 & 9.616 & 124.761 & 125.789 & 0.226 \\
$5^{6}$ & $\mathcal{N} \mathcal{F}$ & 150.315 & 11.538 & 149.833 & 150.693 & 0.186 \\
$5^{6}$ & $\mathcal{R W}$ & 1302.779 & 100.000 & 1298.263 & 1306.771 & 1.798 \\
$5^{6}$ & $\mathcal{H}_{1}$ & 973.049 & 74.690 & 970.787 & 975.678 & 1.110 \\
$5^{6}$ & $\mathcal{H}_{2}$ & 506.337 & 38.866 & 505.410 & 507.813 & 0.446 \\
$5^{6}$ & $\mathcal{H}_{3}$ & 225.869 & 17.337 & 225.381 & 226.277 & 0.180 \\
\hline$n$ & $\mathcal{F}$ & 170.164 & 2.397 & 169.353 & 171.078 & 0.408 \\
$n$ & $\mathcal{N F}$ & 300.069 & 4.227 & 299.385 & 300.726 & 0.274 \\
$n$ & $\mathcal{R} \mathcal{W}$ & 7098.130 & 100.000 & 7063.073 & 7121.211 & 12.755 \\
$n$ & $\mathcal{H}_{1}$ & 5816.386 & 81.943 & 5773.399 & 5849.654 & 16.886 \\
$n$ & $\mathcal{H}_{2}$ & 1904.744 & 26.834 & 1895.577 & 1911.731 & 4.032 \\
$n$ & $\mathcal{H}_{3}$ & 809.288 & 11.401 & 806.836 & 812.015 & 1.328 \\
\hline
\end{tabular}

algorithms have bad performance according to Mean. $\mathcal{R W}$ is the best algorithm; $\mathcal{F}$ and $\mathcal{N} \mathcal{F}$ are the worst algorithms. Our hybrid algorithms are between these two extreme behaviours.

f) Summary: For binomial and d-regular graphs, all algorithms have close performance. Therefore normalized flooding is a good choice in this case as it is faster than other
TABLE XII

PERCENTAGE OF DISCOVERED NODES BY THE $k$-WALKERS ALGORITHM TO THE HYBRID ALGORITHM FOR POWER-LAW GRAPHS

\begin{tabular}{|l|r|r|r|c|}
\hline Parameters & \multicolumn{1}{|c|}{ Mean } & Minimum & Maximum & \multicolumn{1}{c|}{$\sigma$} \\
\hline$\lambda=2.1$, average degree 5 & 75.469 & 3.490 & 100.000 & 33.948 \\
$\lambda=2.1$, average degree 10 & 77.399 & 3.026 & 100.000 & 32.857 \\
$\lambda=2.7$, average degree 5 & 71.490 & 0.146 & 100.000 & 38.505 \\
$\lambda=2.7$, average degree 10 & 75.945 & 0.448 & 100.000 & 35.159 \\
\hline
\end{tabular}

TABLE XIII

PERCENTAGE OF DISCOVERED NODES BY THE $k$-WALKERS ALGORITHM TO THE HYBRID ALGORITHM FOR CLUSTERED TOPOLOGIES

\begin{tabular}{|l|r|r|r|c|}
\hline Clusters & \multicolumn{1}{|c|}{ Mean } & Minimum & Maximum & $\sigma$ \\
\hline$G_{100,1 / 5}$ & 99.828 & 98.638 & 100.173 & 0.407 \\
$G_{100,1 / 2}$ & 99.970 & 99.689 & 100.199 & 0.110 \\
Cliques & 99.966 & 99.699 & 100.198 & 0.114 \\
\hline
\end{tabular}

algorithms. This means that it takes less time on average to discover a node. For power-law graphs and large number of queries flooding does not have good performance and our hybrid algorithm outperforms it. For clustered topologies flooding and normalized flooding are ineffective and random walk has the best performance. The hybrid algorithms' behaviour mediates between flooding and random walk.

\section{Hybrid Algorithm vs. $k$-Walkers}

Our proposed hybrid algorithm uses flooding to find $k$ distinct outer nodes and then starts $k$ independent walkers from those nodes. An alternative is to initiate $k$ independent walkers from the origin, i.e., the $k$-walkers algorithm. Intuitively, the walkers have less overlap in the hybrid algorithm. Therefore the hybrid algorithm increases the dispersion.

We conducted several experiments to compare the performance of these two algorithms on power-law and clustered topologies. We considered different number of walkers and compared the percentage of discovered nodes by the two algorithms with the same number of messages. For powerlaw graphs, the hybrid algorithm outperforms $k$-walkers most of the time. We considered 158 different values for $k$ from 1 to 30000 . Table XII shows the average, minimum, maximum, and standard deviation of the percentage of nodes discovered by the $k$-walkers algorithm to the hybrid algorithm for powerlaw graphs.

According to these results, the $k$-walkers algorithm never outperforms the hybrid algorithm and on average discovers around $3 / 4$ as many nodes as the hybrid algorithm. The minimum percentage for all these settings is achieved for $k=25500$. For $k=1$, the algorithms are equivalent and the percentage is 100 . For large number of walkers, the hybrid algorithm is much more effective than the $k$-walkers algorithm.

For the clustered topologies, they have similar performance and each of them outperforms the other on some settings. We considered 69 distinct values for $k$ from 1 to 1000. The corresponding experimental results are shown in Table XIII. On average, the hybrid algorithm outperforms the $k$-walkers algorithm, but their performance is very close. 


\section{CONCLUSIONS}

In this paper we analyzed the performance of several search algorithms on unstructured peer-to-peer networks. We introduced a new hybrid algorithm that combines flooding with random walk. It uses flooding to find sufficient, say $k$, outer nodes and then starts $k$ independent random walks from those nodes. We compared this algorithm with classic search algorithms such as flooding and random walk, on binomial random graphs, regular random graphs, power-law graphs, and clustered topologies. These are the common topologies for peer-to-peer networks. Our experiments showed that

- For binomial random graphs and regular random graphs, all algorithms have similar behaviour.

- For power-law graphs, flooding is effective for small number of messages, but for large number of messages our hybrid algorithm outperforms it.

- Flooding is ineffective for clustered topologies in which random walk is the best algorithm. For these topologies, our hybrid algorithm provides a compromise between flooding and random walk.

We also compared the proposed hybrid algorithm with the $k$-walker algorithm on power-law and clustered topologies. The $k$-walker algorithm starts $k$ independent walks from the origin. Our experiments showed that while they have similar performance on clustered topologies, the hybrid algorithm has much better performance on power-law graphs.

\section{ACKNOWLEDGMENTS}

Part of this work was done while the second author was visiting the Caesarea Rothschild Institute at the University of Haifa, under the auspices of the University of Waterloo-Haifa International Experience Program.

This work was made possible by the facilities of

- the Shared Hierarchical Academic Research Computing Network SHARCNET (www.sharcnet.ca): 8,082 CPUs,

- the Atlantic Computational Excellence Network ACEnet (www.ace-net.ca): 412 CPUs,

- the Department of Combinatorics and Optimization, University of Waterloo (www.math.uwaterloo.ca/CandO_Dept): 80 CPUs.

\section{REFERENCES}

[1] A. Bonato. A survey of models of the web graph. In Proceedings of the 1st Workshop on Combinatorial and Algorithmic Aspects of Networking (CAAN '04), pages 159-172, 2004.

[2] A. Broder, R. Kumar, F. Maghoul, P. Raghavan, S. Rajagopalan, R. Stata, A. Tomkins, and J. Wiener. Graph structure in the web. In Proceedings of the 9th International World Wide Web Conference, pages 309-320, 2000.

[3] E. Cohen and S. Shenker. Replication strategies in unstructured peer-topeer networks. Proceedings of the ACM SIGCOMM 2002 Conference on Applications, Technologies, Architectures, and Protocols for Computer Communications (SIGCOMM '02), 32(4):177-190, 2002.

[4] C. Gkantsidis, M. Mihail, and A. Saberi. Random walks in peer-topeer networks. In Proceedings of the 23rd Annual Joint Conference of the IEEE Computer and Communications Societies (INFOCOM 'O4), volume 1, pages 120-130, 2004.
[5] C. Gkantsidis, M. Mihail, and A. Saberi. Hybrid search schemes for unstructured peer-to-peer networks. In Proceedings of the 24th Annual Joint Conference of the IEEE Computer and Communications Societies (INFOCOM '05), volume 3, pages 1526-1537, 2005.

[6] S. Janson, T. Łuczak, and A. Ruciński. Random Graphs. WileyInterscience Series in Discrete Mathematics and Optimization. John Wiley \& Sons, 2000

[7] T. Łuczak and P. Prałat. Protean graphs. Internet Mathematics, 3(1):2140, 2006.

[8] Q. Lv, P. Cao, E. Cohen, K. Li, and S. Shenker. Search and replication in unstructured Peer-to-Peer networks. In Proceedings of the 2002 International Conference on Measurement and Modeling of Computer Systems (SIGMETRICS-02), volume 30, 1, pages 258-259, 2002.

[9] P. Prałat. A note on the diameter of protean graphs. Discrete Mathematics. to appear.

[10] P. Prałat and N. Wormald. Growing protean graphs. Internet Mathematics. to appear.

[11] A. Ruciński and N. C. Wormald. Random graph processes with degree restrictions. Combinatorics, Probability \& Computing, 1:169-180, 1992.

[12] N. C. Wormald. Models of random regular graphs. In Surveys in Combinatorics, 1993, Walker (Ed.), London Mathematical Society Lecture Note Series 187, Cambridge University Press, volume 276, pages 239-298. 1999. 